\title{
Scheduling Operator Attention for Multi-Robot Control
}

\author{
Shih-Yi Chien, Michael Lewis \\ School of Information Sciences \\ 135 North Bellefield Avenue \\ Pittsburgh, PA, 15260 U.S.A. \\ 011-412-624-9426 \\ shc56@pitt.edu, \\ ml@sis.pitt.edu
}

\author{
Siddharth Mehrotra \\ Agents Dynamics Inc. \\ 6370 Phillips Avenue \\ Pittsburgh, PA, 15217 U.S.A. \\ 011-412-951-4439 \\ siddharthmehrotra1 1 \\ @gmail.com
}

\author{
Nathan Brooks, Katia Sycara \\ Robotics Institute \\ Carnegie Mellon University \\ Pittsburgh, PA 15213 U.S.A. \\ 011-412-268-8825 \\ nbb@andrew.cmu.edu, \\ katia@cs.cmu.edu
}

\begin{abstract}
A wide class of multirobot control tasks involves operator interactions with individual robots. Where the robots' actions are independent, as for example in some foraging tasks, the operator can interact with robots sequentially in a round robin fashion. If the need for interaction can be detected by the robot through selfreflection, the robot could communicate its need for interaction to the operator. The resulting human-robot system would form a queuing system in which the operator is the server and the queue of robots requesting interaction, the jobs. As a queuing system, performance could be optimized using standard techniques, providing the operator's attention could be appropriately directed. An earlier study found that HumanRobot Interaction (HRI) performance was improved by communicating requests for interaction to the operator, however, a first-in-first-out (FIFO) aid showing a single request at a time led to poorer performance than one showing the entire (Open) queue. The current experiment compared Open-queue and FIFO conditions from the first experiment with a Priority-queue using a shortest job first (SJF) discipline known to maximize throughput. Performance in the Priorityqueue condition was statistically indistinguishable from the best performance for all measures except those for missed victims where it was intermediate between FIFO (best) and Open-queue. Both of the other conditions produced poorest performance on some measures. The results suggest that operator attention can be effectively scheduled allowing the use of scheduling algorithms to improve the efficiency of HRI.
\end{abstract}

\section{INTRODUCTION}

$\mathrm{I}$ $\mathrm{n}$ the simplest case of multirobot control, an operator controls multiple independent robots interacting with each as needed. A foraging task [1] in which each robot searches its own region would be of this category. Control performance at such tasks can be characterized by the average demand of each robot on human attention [2]. Such operator interactions with a robot might be described as a sequence of control episodes in which an operator interacts with the robot for period of time (interaction time, IT) raising its performance above some upper threshold (UT) after which the robot is neglected for a period of time (neglect time, NT) until its performance deteriorates below a lower threshold (LT) when the operator must again interact with it. In practice the operator's task is even more complex. Humans are additionally included in robotic systems to perform tasks the automation cannot. The most common of these tasks is searching for targets in noisy displays such as remote video or aerial imagery.

Research in robot self-reflection [3] has progressed to the point that it is plausible to presume robots capable of reporting their own off normal conditions such as an inability to move or unsafe attitude. By focusing the operator's attention on robots needing interaction rather than requiring the operator to monitor for the failures, time spent monitoring can be eliminated increasing the number of robots that can be serviced over this interval. With robots informing the operator of their need for interaction the human-robot system becomes a queuing system in which the operator acts as the server and robot interaction requests as jobs. Using operations research methods the performance of such a queuing system might be further improved by prioritization of jobs or adjustment of service levels [4] to match current conditions. Deriving full benefit from such aiding, however, would require the ability to focus an operator's attention on a particular robot.

The prior experiment [5] compared a control condition without requests for interaction with experimental conditions in which either a single request (FIFO-queue) or all requests (Open-queue) were shown to the operator. Because ITs for recovery were the same across failures there should have been no difference in performance between operators presented with robots needing service individually or as a group. Instead, we found consistent advantages for the Open-queue condition that showed all failures. There are two plausible explanations for this finding: 1) despite the apparent equivalence of repairs, operators were able to take advantage of situational differences involving things such as proximity to targets or other robots to choose robots for repair that led to higher overall performance or 2) the system's designation of the robot to be serviced next seemed too restrictive to operators who therefore did not consistently follow its advice. Direction of operators at this level of specificity has often met with resistance [2], [6]. Because the FIFO queue always presented a robot in an appropriately failed state, however, the aid was errorless and not subject to the usual manipulations of trust [7]. 


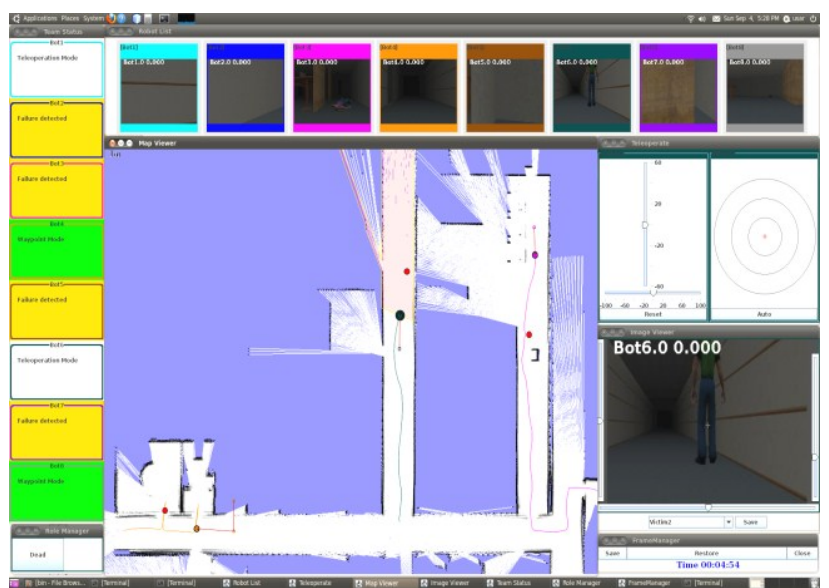

Figure 1. Alarm condition display (Open-Queue).

We found directed attention inferior to undirected alarms under conditions in which there should have been no difference for compliant operators. The possibility, however, remained that directed attention might improve performance under conditions in which compliance would produce superior performance to random selection from among the alarming robots. To examine the effects of attention direction through a prioritized queue, heterogeneous errors with different recovery times were introduced. This allowed a Priority-queue following the throughput maximizing SJF (Shortest Job First) discipline [8]. If operators strictly followed the recommendations of the Priority-queue they should achieve the best attainable performance. We therefore hypothesized that a Priorityqueue performance should be equal or greater than that in the Open-queue condition if the operator complies with its recommendations. In other words, the operator attention could be optimized based on an effective scheduling approach. Additionally, the FIFO-queue by contrast employs a suboptimal discipline and should lead to poorer performance for a strictly complying operator.

\section{METHODS}

\section{A. USARSim and MrCS}

The experiment was conducted using the USARSim robotic simulation with 8 simulated Pioneer P3-AT robots performing an Urban Search and Rescue (USAR) foraging task. USARSim is a high-fidelity simulation of USAR robots and environments developed as a research tool for the study of human-robot interaction (HRI) and multi-robot coordination. USARSim supports HRI by accurately rendering user interface elements (particularly camera video), accurately representing robot automation and behavior, and accurately representing the remote environment that links the operator's awareness with the robot's behaviors. USARSim uses Epic Games' UnrealEngine3 to provide a high fidelity simulator at low cost and also serves as the basis for the Virtual Robots Competition of the RoboCup Rescue League. Other sensors including sonar and audio are also accurately modeled.

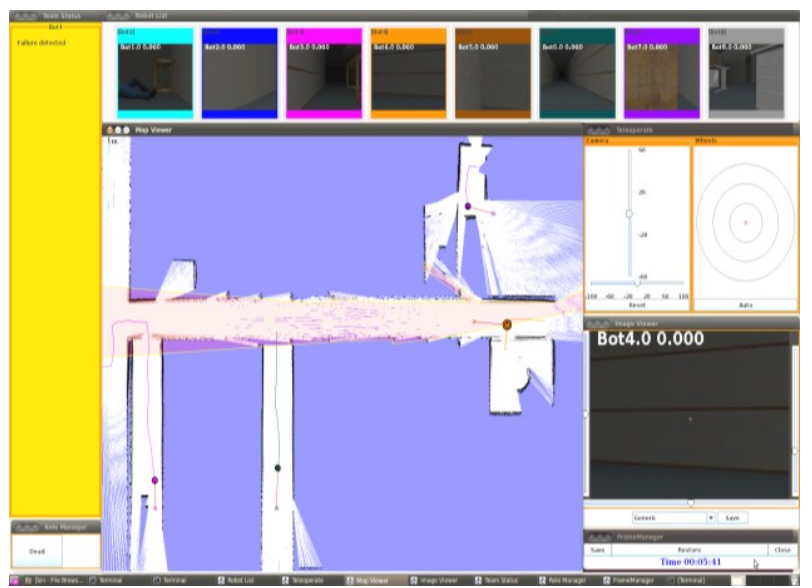

Figure 2. Decision aid condition display (FIFO-/Priority-Queue).

MrCS (Multi-robot Control System), a multi-robot communications and control infrastructure with accompanying user interface, developed for experiments in multirobot control and RoboCup competition [9] was used in this experiment. MrCS provides facilities for starting and controlling robots in the simulation, displaying multiple camera and laser output, and supporting inter-robot communication through Machinetta which is a distributed multi-agent coordination infrastructure.

Figure 1 shows the MrCS user interface in alarm condition. Thumbnails of robot camera feeds are shown on the top, a video feed of interest in the bottom right. A GUI element in the middle right allows teleoperation and camera pan and tilt. Current locations and paths of the robots are shown on the Map Viewer (middle) which also allows operators to mark victims. The team status window (left) for the Open-queue condition shows each robot's current status and briefly summarizes any problem. Green indicates the robot is in autonomous condition and functioning safely, yellow indicates an abnormal condition, such as stuck at a corner. When a robot is manually controlled, its tile turns white. The operator selects the robot to be controlled from either the team status window or camera thumbnail.

Figure 2 shows the team status window for the forced queue (FIFO or SJF) conditions in which robots in abnormal states are presented one at a time. Additional alarms can only be reviewed after the presenting problem is resolved. To avoid "clogging" the status window with an unrecoverable failure, operators have an alternative in the Dead button (bottom left). Once switched off, the robot will stop reporting and no longer be scheduled.

\section{B. USAR Foraging task}

When an operator detects a victim in a thumbnail, a complex sequence of actions is initiated. The operator first needs to identify the robot and select it to see the camera view in a larger window and to gain the ability to stop or teleoperate the robot. After the user has successfully selected a robot, it must be located on the map by matching the window border color or numerical label. Next the operator must determine the orientation of the robot and its 
camera using cues such as prior direction of motion and matching landmarks between camera and map views. To gain this information the operator may choose to teleoperate the selected robot to locate it on the map, determine its orientation through observing the direction of movement, or simply to get a better viewing angle. The operator must then estimate the location on the map corresponding to the victim in the camera view. If "another" victim is marked nearby, the operator must decide whether the victim she is preparing to mark has already been recorded on the map.

Detecting and restoring a failed robot follow a similar time course: identifying the failed robot on the map and selecting it, then teleoperating it to its next waypoint where the automation can resume control.

\section{Types of Failures}

Recoverable failures were categorized into 4 major types, based on the data for commonly occurring non terminal and field repairable failures for the Pioneer P3-AT [10]. Two of these, camera and map failures, involve loss of display due to communication difficulties. The third, teleoperation lag is a control problem found by Ferrell [11] and Sheridan [12] to significantly degrade operator performance. The fourth, "stuck", is a common condition in which a robot becomes entangled with obstacles. To resolve encountered failures, the operator needed to manually guide the robot from its current location to the next waypoint. Because each of the failure types imposed different difficulties for recovery, they took varying amounts of time to resolve. In order to estimate typical resolution times for different failures, a pretest using 10 participants was conducted.

TABLE 1. FOUR TYPES OF FAILURES OCCURRED IN THE STUDY.

\begin{tabular}{|c|l|}
\hline Failure & \multicolumn{1}{|c|}{ Description } \\
\hline Stuck & $\begin{array}{l}\text { Robot was stopped by approaching } \\
\text { obstacles }\end{array}$ \\
\hline $\begin{array}{c}\text { Teleoperation } \\
\text { Lagged }\end{array}$ & $\begin{array}{l}\text { Robot executed operator's command } \\
\text { with 2 3 seconds delay }\end{array}$ \\
\hline $\begin{array}{c}\text { Camera } \\
\text { Sensor Failed }\end{array}$ & $\begin{array}{l}\text { Robot's video feed will be frozen right } \\
\text { before the failure happened }\end{array}$ \\
\hline $\begin{array}{c}\text { Map Viewer } \\
\text { Failed }\end{array}$ & $\begin{array}{l}\text { Robot's position on the map viewer } \\
\text { will be unable to update }\end{array}$ \\
\hline
\end{tabular}

In the training session, participants practiced control operations for different types of failures for 5 minutes each. Participants were instructed that their goal was to resolve failures by teleoperating to the next indicated waypoint as rapidly as possible. To avoid unrelated delays, such as those associated with switching attention among robots, participants controlled a single designated robot at a time. Because teleoperating the robot to its next waypoint was most easily accomplished by locating both on the map, loss of map indication proved to be the lengthiest failure to repair. The stuck condition which required extensive manual maneuvering and the camera failure that made obstacle avoidance more difficult were the easiest to overcome, with teleoperation delay falling in the middle, figure 3. This ordering of estimated interaction times allowed failures to be presented to the operator in a priority queue following a shortest job first (SJF) discipline, known to maximize throughput [8].

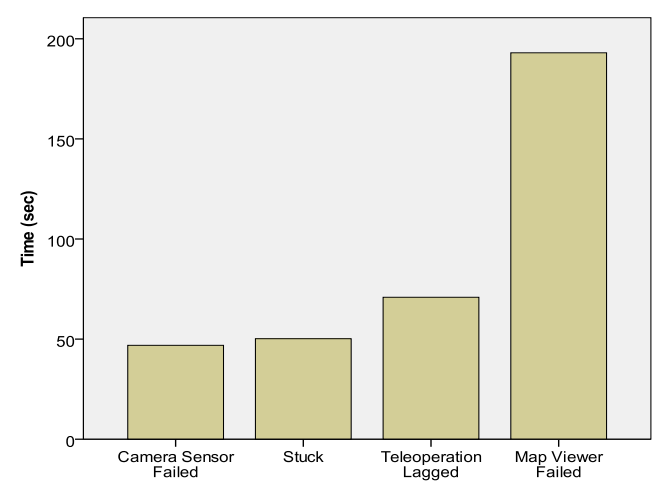

Figure 3. Interaction time for failures.

\section{Experimental Conditions}

The selected USAR environment was an office like hall with many rooms full of obstacles like chairs and desks. Victims were evenly distributed within the environment. Maps were rotated by $90^{\circ}$ and each robot entered the environment from different locations on each trial. Because the laser map is built up slowly as the environment is explored and the office like environment provides few distinctive landmarks, there was little opportunity for participants to benefit from prior exposure to the environment. A team size of 8 robots found by [13] to yield the highest performance at the USAR task was used in the experiment. Robots followed predefined paths of waypoints, similar to paths generated by an autonomous path planner [14] to explore the map. All robots traveled paths of the same distance with ten visible victims and four failures in each designed path. Upon reaching a failure point the robot experienced a failure, such as becoming entangled with a chair or sensor failures. The operator then needed to assume manual control to teleoperate the robot out of its predicament to its next waypoint where communications could be reestablished for lost camera feed or control and autonomous exploration resumed. The experiment followed a three condition repeated measures design comparing the MrCS augmented by alarm panels with two decision aid approaches. Experimental conditions were fully counterbalanced, for map orientations, robots' starting points, and displays, in which 5 participants were run in each of the six cells.

\section{E. Participants and Procedure}

The experiment followed a within-group design. 30 paid participants were recruited from the University of Pittsburgh community balanced among conditions for gender. None had prior experience with robot control although most were frequent computer users. Participants 
read standard instructions on how to control robots via MrCS. In the following 20 minute training session, 5 minutes for each type of failure, participants practiced control operations by resolving failures, three times for each type. Participants were encouraged to find and mark at least one victim in the training environment under the guidance of the experimenter. After the training session, participants began the first 15 minute experimental session in which they performed the foraging task controlling 8 robots in their first assigned condition. Participants had been told the main task was to locate victims with detecting and resolving robot failures as a secondary task. At the conclusion of the session, participants were asked to complete the NASA-TLX workload survey [15]. After brief breaks, the next two conditions were run accompanied by repeated workload surveys.

\section{F. Measurements}

To evaluate the effects of different scheduling schemes the following measurements were adopted:

TABLE 2. MEASUREMENT SCALES.

\begin{tabular}{|c|l|}
\hline Category & \multicolumn{1}{|c|}{ Concept } \\
\hline $\begin{array}{c}\text { Victim } \\
\text { Rescued }\end{array}$ & $\begin{array}{l}\text { Primary task: identifying more victims } \\
\text { corresponds to better performance }\end{array}$ \\
\hline $\begin{array}{c}\text { Distance } \\
\text { Traveled }\end{array}$ & $\begin{array}{l}\text { Larger explored areas could result in } \\
\text { greater opportunities to find more victims }\end{array}$ \\
\hline $\begin{array}{c}\text { Event } \\
\text { Timeline }\end{array}$ & $\begin{array}{l}\text { Characterizing the operators' allocation of } \\
\text { attention in controlling robots, shorter } \\
\text { times indicate better performance }\end{array}$ \\
\hline $\begin{array}{c}\text { Failures } \\
\text { Resolved }\end{array}$ & $\begin{array}{l}\text { Secondary task: indicating the operators' } \\
\text { efficiency in resolving robot failures }\end{array}$ \\
\hline Workload \\
Survey
\end{tabular} \begin{tabular}{l}
$\begin{array}{l}\text { Subjective report of cognitive workload, } \\
\text { lower workload indicates better system } \\
\text { performance }\end{array}$ \\
\hline
\end{tabular}

\section{RESULTS}

\section{A. Victims Found \& Distance Traveled}

No difference was found for the number of victims identified $(\mathrm{F}(2,58)=.110, \mathrm{p}=.896)$. Each victim marking was compared to ground truth to determine whether there was actually a victim near the location. If a mark was made further than 2 meters away from any victim or multiple marks for a single victim were found, the marks were counted as false positives. The number of false positives showed a main effect for queue condition $(\mathrm{F}(2,58)=4.637$, $\mathrm{p}=.014$ ), figure 4. A pairwise T-test found a significant difference between Open-queue (1.13 false) and FIFOqueue ( 2 false) conditions $(\mathrm{p}=.030)$, as well as a difference between Priority-queue (1.2 false) and FIFO-queue $(p=.012)$. No effects were found between Open-queue and Priority-queue.

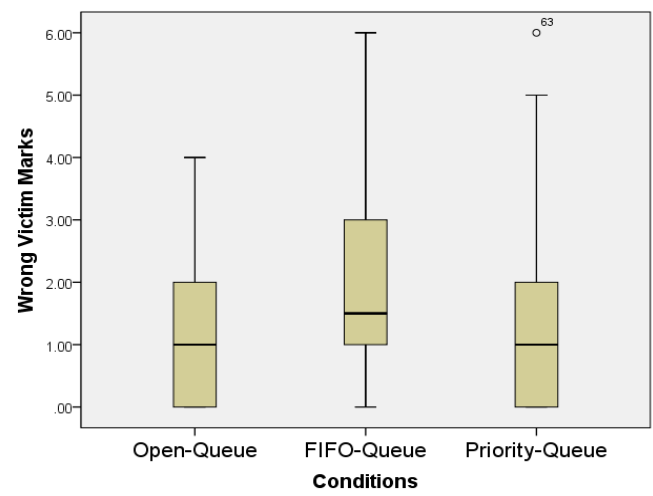

Figure 4. Number of wrong marks (false positive).

Unmarked victims that had appeared within a robot's FOV (field of view) without being marked were counted as false negatives (misses). Operators in the Open-queue condition missed the most victims (15) and the FIFO-queue was the lowest (11) with the Priority-queue falling in between (13), as shown on figure 5. A repeated measures ANOVA shows a main effect among queue conditions, $\mathrm{F}(2,58)=20.5, \mathrm{p}<.001$. Pairwise T-tests revealed significant differences between Open-queue and FIFO-queue $(p<.001)$, Open-queue and Priority-queue $(\mathrm{p}=.006)$, and Priorityqueue and FIFO-queue condition ( $\mathrm{p}=.003)$.

No difference was found for the distance traveled $(\mathrm{F}(2,58)=1.73, \mathrm{p}=.186)$ although Open-queue $(321 \mathrm{~m})$ appears slightly better than FIFO-queue $(293 \mathrm{~m})$ with Priority-queue again in the middle $(310 \mathrm{~m})$.

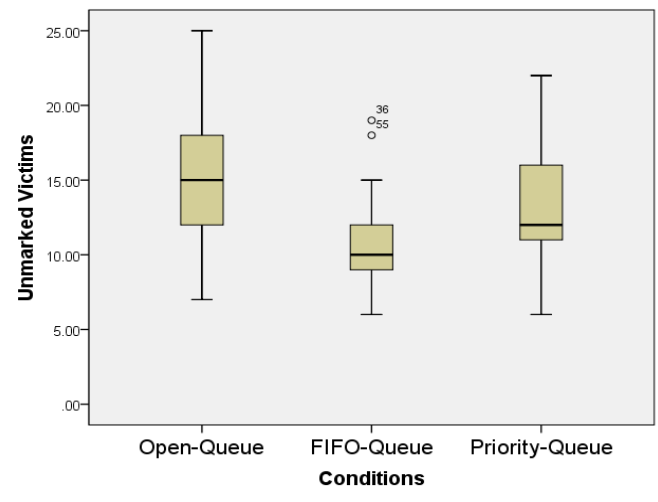

Figure 5. Number of unmarked victims (false negative).

Since avoiding missed targets is crucial to many foraging tasks, missing victims might have resulted simply from the greater opportunity afforded by exploring larger areas. To examine this possibility we tested the adjusted measure missed-victims per distance traveled Distance traveled is defined by the sum of the distances each robot has navigated through the environment during the experiment. As seen in figure 6, the results show operators in the Open-queue condition missed the most victims per region explored (.49) and the FIFO-queue the fewest (.40) with the Priority-queue falling in between (.45) . Again, a main effect was found among conditions $(\mathrm{F}(2,58)=11.238$, $\mathrm{p}<.001)$. Pair-wise $\mathrm{T}$-test revealed significant difference 
between Open-queue and FIFO-queue condition $(\mathrm{p}<.001)$, Open-queue and Priority-queue condition $(\mathrm{p}=.046)$, and Priority-queue and FIFO-queue condition ( $\mathrm{p}=.015)$.

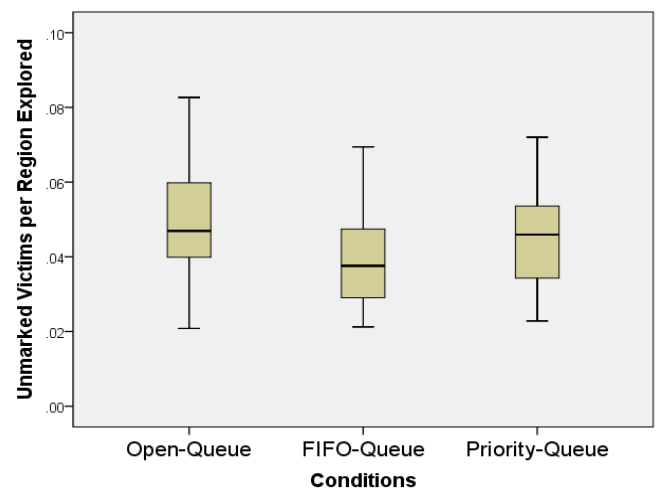

Figure 6. Unmarked victims per region explored.

\section{B. Event Timelines}

Neglect time (NT) and latency in responding to failures are indicators of the operator performance. Long NTs can indicate that some robots may have been ignored while latency in responding to failures can suggest noncompliance with assistance requests or heavy workload at other parts of the task. NT $(\mathrm{F}(2,58)=1.66, \mathrm{p}=.20)$ and the latency in responding to failure $(\mathrm{F}(2,58)=1.75, \mathrm{p}=.183)$ were not significantly different among the three conditions. Pairwise T-tests found no difference between Open-queue and FIFO queue in either Neglect Time, figure $7(\mathrm{p}=.086)$ or fault detection time, figure $8(\mathrm{p}=.079)$. A prior study [5], however, found an advantage for Open queue over FIFO queue for response latency under similar conditions.

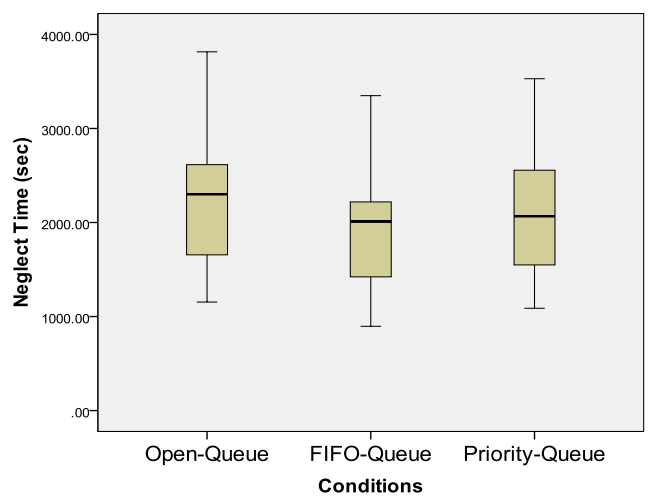

Figure 7. Neglect time

The time to service failed robots, measured as the time between selecting the robot and resolving its problem again showed no difference among conditions $(\mathrm{F}(2,58)=.579$, $\mathrm{p}=.507$ ), which suggests the types of pre-designed failures were well distributed among three conditions. Overall, FIFO-queue appears slightly worse in the above three measurements.

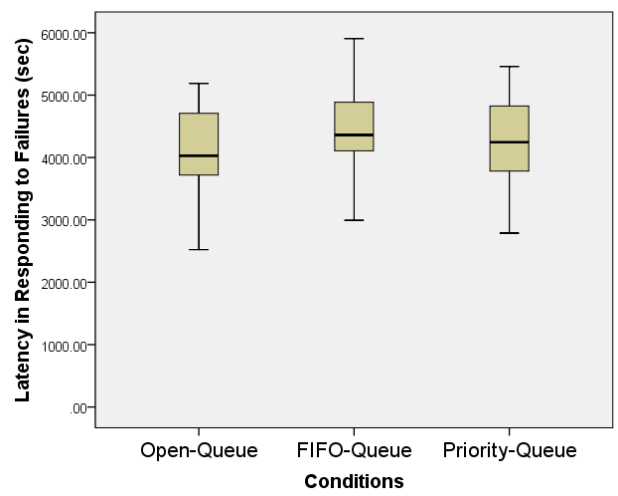

Figure 8. Latency in responding to failures.

Select-to-Mark, is defined by the interval between selecting a robot with a victim in view and marking that victim on the map. Select to mark times can be interpreted as a measure of situation awareness (SA) because they require the operator to orient and interpret the environment. A repeated measures ANOVA shows a significant difference among conditions $(\mathrm{F}(2,58)=5.413, \mathrm{p}=.011)$. Operators in the FIFO-queue condition took the longest time $(583 \mathrm{sec})$ and the Open-queue was the shortest $(389$ $\mathrm{sec})$ with the Priority-queue falling in between $(478 \mathrm{sec})$, figure 9. A pairwise $\mathrm{T}$-test showed a significant difference between Open-queue and FIFO-queue conditions ( $p=.002)$, and a marginal difference between Open-queue and Priority-queue $(\mathrm{p}=.061)$.

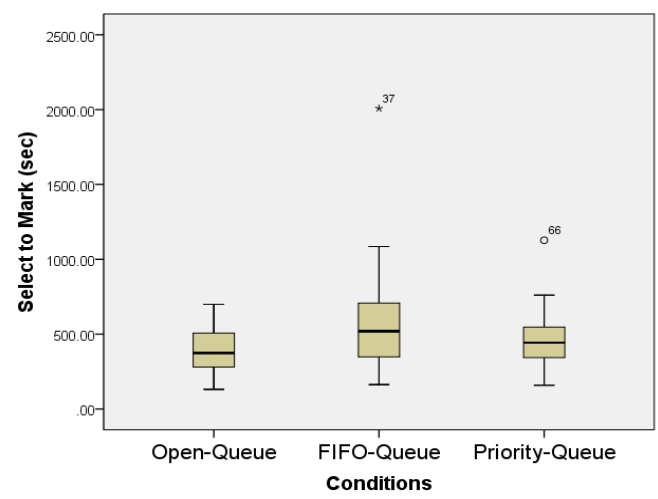

Figure 9. Accumulated Select to Mark time.

\section{Failures Resolved}

The operator must successfully teleoperate the stopped robot from its current location to the next predefined waypoint to resolve a failure. A repeated measures ANOVA showed a significant difference for the count of resolved failures among experimental conditions $(\mathrm{F}(2,58)=5.5$, $\mathrm{p}=.006$ ), figure 10. Participants in the Open-queue condition solved the most failures (17.8), which was significantly more than FIFO-queue $(\mathrm{p}=.003)$. A pairwise T-test also revealed a difference between Priority-queue, 17 failures, and FIFO-queue, 15.7 failures, $(p=.057)$. 


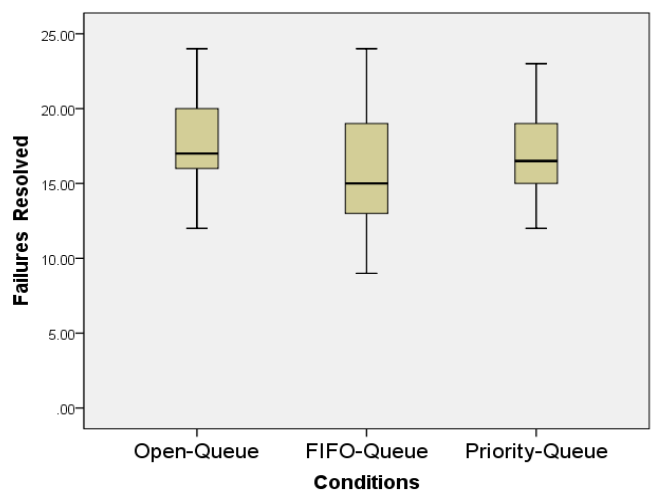

Figure 10. Number of failures resolved.

\section{Workload}

The full-scale NASA-TLX workload measure found no advantage among conditions. To examine effects related to the highly prescriptive aiding in the FIFO and Priority queue, we analyzed the frustration scale separately.

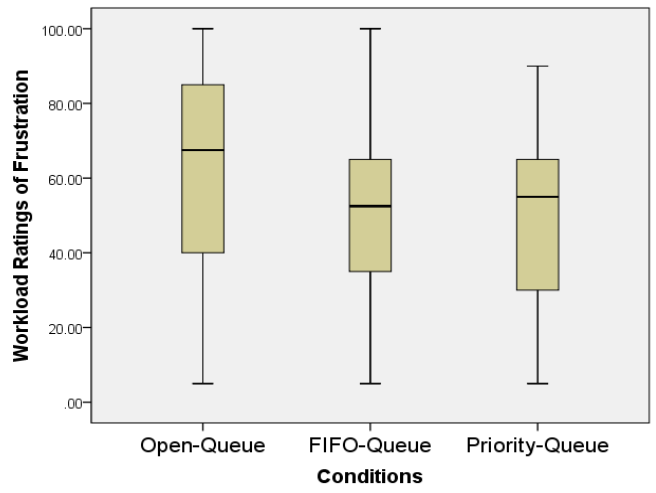

Figure 11. Subjective workload ratings of Frustration.

Repeated measures ANOVA showed a significant difference $(\mathrm{F}(2,58)=5.159, \mathrm{p}=.009)$, figure 11. Pairwise Ttests revealed differences between Open-queue and FIFOqueue $(\mathrm{p}=.038)$ and between Open-queue and Priorityqueue $(\mathrm{p}=.004)$.

\section{DISCUSSION}

In a previous study [5] we found that alerting operators to robots in need of interaction improved performance along a number of dimensions. The earlier study compared a control condition without alerting with experimental conditions corresponding to the Open-queue and FIFOqueue conditions of the current experiment. While alerting was beneficial, the earlier study found FIFO-queue which directed the operator to service a particular robot was less effective than the Open-queue which allowed the operator to choose. This advantage could be explained either by superiority of strategies of Open-queue operators when allowed choice or operator difficulties in complying with an aide that prescribed the robot to be serviced.

\section{A. Hypothesis Validation}

Our present study reflects the effects of operator attention allocation using various forms of alerting assistance. The results roughly support the hypothesis that operator attention can be effectively directed to interaction with individual robots. Open-queue performed slightly better than Priority-queue on false positives, distance traveled, and failures resolved, but only for select-to-mark times did the difference approach significance. For the primary task of marking victims, FIFO participants proved slightly better, however, SJF participants were significantly superior to Open-queue users yielding a balanced performance which was never poorest. The above results may be due to the differences in allocation of attention. Within limited cognitive capacity of processing information, operators have to selectively dedicate attention to any of the "wanted" targets and filter out the irrelevant information simultaneously [16]. Open-queue operators must devote time and attention to monitoring and selection of robots for servicing as well as the interaction leaving less available for the victim monitoring and marking tasks; whereas operators in the forced queue (Priority-/FIFO-Queue) conditions, by contrast, do not have to compete with monitoring and selecting robots to service leaving more resources available for victim-related tasks, which leads to the reversed results in unmarked victims among three conditions.

The FIFO-queue condition which directed operator attention suboptimally also led to the greatest loss of SA as reflected in its longest Select-to-Mark victims times and lowest marking accuracy. This may have been exacerbated by the FIFO discipline which did not distinguish between distracting recoveries such as loss of track on map and brief interventions such as maneuvering around an obstacle. For the Priority-queue, the SJF discipline had not only the advantage of allowing operators to work primarily on briefer interventions thereby preserving SA, but by clustering similar types of failures increased opportunities for reducing the cost to switch between recovery strategies and sharing the similar cognitive procedures among failures. However, the Priority-queue operators may have simply devoted more of their time and attention to robot requests than operators using the less efficient FIFO because of their greater payoff, which could be observed from the higher rate of unmarked victims.

\section{B. User Compliance in Forced Queue Conditions}

Difficulties in obtaining compliance have frequently been reported for highly prescriptive decision aids [17]. Crandall et al. [18] recently reported problems in directing operator attention to particular classes of tasks at a multirobot control task similar to the one reported here. A more general finding is that even minor conflicts between an operator's intentions and an aid's suggestions can lead to disuse [17]. This experiment provided a clear advantage to participants complying with the Priority-queue and a relative disadvantage to those complying with the FIFO- 
queue. Participants in the Open-queue condition who chose to follow the SJF discipline (service map failures last) could potentially match the performance of Priority-queue users. Results generally support this ordering with FIFO participants resolving the fewest failures (secondary task) while identifying the most victims (primary task); however, there were no main effects for the number of victims found. SJF participants had a more balanced performance finding marginally fewer victims but matching Open-queue performance on resolving failures.

In forced queue conditions operators receive an explicit recommendation for the robot to assist. Under extreme stress or time pressured tasks, humans tend to defer to automation and rely on the system for making decisions [19]. This increased compliance under high workload could be especially beneficial to system performance where optimal strategies such as SJF can be used to steer operator attention. Although automated aids can reduce decisional load, they carry little additional information about other robots in need of assistance or the general state of the system [20], [21]. Operators therefore need to regain SA every time they switch to serve a new robot. While working from a forced queue, operators must match the alarmed robots to the thumbnails and/or maps, which could increase the cost in switching attention among failures and robots.

\section{User Perception of Workload}

The NASA-TLX survey revealed differences among conditions where the Open-queue was judged significantly more frustrating than either the SJF or FIFO queues. Potential causes for frustration in the Open-queue condition reported in debriefings were:

1) Endless failures: participants felt distracted with so many robots requiring attention

2) Showing all failed robots led participants to feel more pressure than with only one robot in the queue.

3) Under overload conditions, participants wanted to rely on automatic recommendations, as provided by the FIFO-/SJF-queue.

The study results are promising for the prospects of improving HRI performance through scheduling operator attention. The improvement of performance in queuing discipline shows that forced queue aiding can be effectively used by operators and might even lead to superior performance under more complex conditions where choice among robot requests becomes more difficult.

\section{ACKNOWLEDGMENTS}

This research has been sponsored in part by AFOSR FA955008-10356 and ONR Grant N0001409-10680.

\section{REFERENCES}

[1] Y. U. Cao, A. S. Fukunaga, and A. Kahng, "Cooperative mobile robotics: antecedents and directions," Autonomous Robots, vol. 4, no. 1, pp. 7-27, 1997.

[2] J. W. Crandall, M. A. Goodrich, D. R. Olsen, and C. W. Nielsen, "Validating Human-Robot Interaction Schemes in Multitasking Environments," IEEE Transactions on Systems Man and
Cybernetics Part A Systems and Humans, vol. 35, no. 4, pp. 438449, 2005.

[3] M. Scheutz and J. Kramer, "Reflection and Reasoning Mechanisms for Failure Detection and Recovery in a Distributed Robotic Architecture for Complex Robots," Components, no. 1, pp. 36993704, 2007.

[4] Y. Xu, T. Dai, K. Sycara, and M. Lewis, "Service Level Differentiation in Multi-robots Control," System, pp. 2224-2230, 2010.

[5] S.-Y. Chien, H. Wang, M. Lewis, S. Mehrotra, and K. Sycara, "Effects of Alarms on Control of Robot Teams," in Proceedings of the Human Factors and Ergonomics Society Annual Meeting, 2011, vol. 55, no. 1, pp. 434-438.

[6] M. A. Goodrich, M. Quigley, and K. Cosenzo, "Task switching and multi-robot teams," MultiRobot Systems From Swarms to Intelligent Automata, vol. III, pp. 185-195, 2005.

[7] J. Lee and K. See, "Trust in automation: designing for appropriate reliance.," Human factors, vol. 46, no. 1, pp. 50-80, Jan. 2004

[8] M. R. Garey, D. S. Johnson, and R. Sethi, "The Complexity of Flowshop and Jobshop Scheduling," Mathematics of Operations Research, vol. 1, no. 2, pp. 117-129, 1976.

[9] S. Carpin, M. Lewis, J. Wang, S. Balakirsky, and C. Scrapper, "Bridging the gap between simulation and reality in urban search and rescue," Robocup 2006 Robot Soccer World Cup X, vol. 4434, pp. 1-12, 2007.

[10] J. Carlson, R. R. Murphy, and A. Nelson, "Follow-up analysis of mobile robot failures," in IEEE International Conference on Robotics and Automation 2004 Proceedings ICRA 04 2004, 2004, vol. 5, no. April, pp. 4987-4994.

[11] W. R. Ferrell, "Remote manipulation with transmission delay," Ieee Transactions On Human Factors In Electronics, vol. HFE-6, no. 1, p. 1,1965

[12] T. B. Sheridan, "Space teleoperation through time delay: review and prognosis," IEEE Transactions on Robotics and Automation, vol. 9, no. 5, pp. 592-606, 1993.

[13] H. Wang, M. Lewis, P. Velagapudi, P. Scerri, and K. Sycara, "How search and its subtasks scale in N robots," Proceedings of the 4th ACMIEEE international conference on Human robot interaction HRI 09, p. 141, 2009.

[14] S. Y. Chien, H. Wang, and M. Lewis, "Human vs . Algorithmic Path Planning for Search and Rescue by Robot Teams," Human Factors, vol. 54, no. 4, pp. 379-383, 2010.

[15] S. G. Hart and L. E. Staveland, "Development of NASA-TLX (Task Load Index): Results of empirical and theoretical research," in Human Mental Workload, vol. 1, P. A. Hancock and N. Meshkati, Eds. North-Holland, 1988, pp. 139-183.

[16] R. Desimone and J. Duncan, "Neural mechanisms of selective visual attention," Annual Review of Neuroscience, vol. 18, no. 1, pp. 193$222,1995$.

[17] A. Kirlik, "Modeling strategic behavior in human-automation interaction: why an 'aid' can (and should) go unused.," Human Factors, vol. 35, no. 2, pp. 221-242, 1993.

[18] J. W. Crandall, M. L. Cummings, M. D. Penna, and P. M. A. D. Jong, "Computing the Effects of Operator Attention Allocation in Human Control of Multiple Robots," IEEE Transactions on Systems Man and Cybernetics Part A Systems and Humans, vol. 41, no. 3, pp. 385-397, 2011.

[19] T. Inagaki, "Adaptive Automation: Sharing and Trading of control," in Handbook of Cognitive Task Design, E. Hollnagel, Ed. 2003.

[20] C. Billings, "Aviation Automation, The search for the HumanCentered Approach," Lawrence Erlbaum, 1997.

[21] R. Parasuraman, T. B. Sheridan, and C. D. Wickens, "A model for types and levels of human interaction with automation.," IEEE transactions on systems, man, and cybernetics. Part A, Systems and humans : a publication of the IEEE Systems, Man, and Cybernetics Society, vol. 30, no. 3, pp. 286-97, May 2000. 